CRYSTALLOGRAPHIC COMMUNICATIONS

ISSN 2056-9890

Received 29 May 2018

Accepted 22 June 2018

Edited by M. Zeller, Purdue University, USA

Keywords: crystal structure; 5-substituted dimethyl isophthalates; $\mathrm{I} \cdots \mathrm{O}=\mathrm{C}$ interaction; $\mathrm{C}-$ $\mathrm{H} \ldots \mathrm{O}$ and $\mathrm{C}-\mathrm{H} \ldots \mathrm{I}$ hydrogen bonding; $\pi-\pi$ stacking.

CCDC references: 780476; 780475

Supporting information: this article has supporting information at journals.iucr.org/e

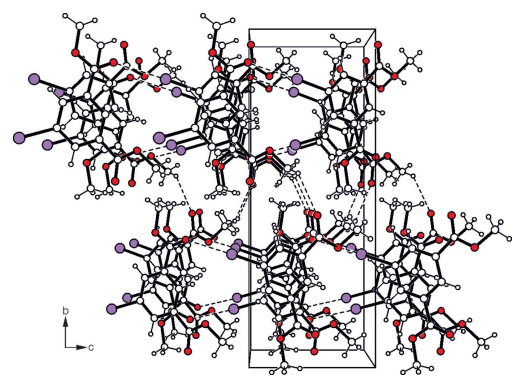

OPEN $\odot$ ACCESS

\section{Crystal structures of dimethyl 5-iodoisophthalate and dimethyl 5-ethynylisophthalate}

\author{
Ines Hauptvogel, Wilhelm Seichter and Edwin Weber*
}

TU Bergakademie Freiberg, Leipziger Str. 29, D-09596 Freiberg/Sachsen, Germany. ${ }^{*}$ Correspondence e-mail: edwin.weber@chemie.tu-freiberg.de

In dimethyl 5-iodoisophthalate, $\mathrm{C}_{10} \mathrm{H}_{9} \mathrm{IO}_{4}$, (I), the planes through the methyl carboxylate moieties are tilted with respect to the benzene ring, whereas the molecular framework of dimethyl 5-ethynylisophthalate, $\mathrm{C}_{12} \mathrm{H}_{10} \mathrm{O}_{4}$, (II), is perfectly planar. The crystal structure of (I) is stabilized by a three-dimensional supramolecular network comprising $\mathrm{C}-\mathrm{H} \cdots \mathrm{O}=\mathrm{C}$ hydrogen bonds, as well as $\mathrm{I} \cdots \mathrm{O}=\mathrm{C}$ interactions. In the crystal of (II), the molecules are connected via $\mathrm{C}-$ $\mathrm{H}_{\text {ethynyl }} \cdots \mathrm{O}=\mathrm{C}$ hydrogen bonds to infinite strands. Moreover, $\pi-\pi$ arene stacking interactions connect the molecular chains into two-dimensional supramolecular aggregates.

\section{Chemical context}

In recent years, the design of solid porous framework materials (MacGillivray, 2010; Furukawa et al., 2013; Eddaoudi et al., 2015) has become a very important topic in the field of supramolecular crystal engineering (Desiraju et al., 2011). Associated with it, so-called linker molecules featuring a geometrically rigid structure frequently being of linear, trigonal or tetrahedral shape and having carboxylic acid functions as terminal groups play a key role in building such systems (Lin et al., 2006; Hausdorf et al., 2009; Zheng et al., 2010). In the course of the synthesis of the respective linkers, the title compounds (I) and (II), both being 5-substituted dimethyl isophthalates, are much used intermediates. However, these compounds are not only synthetically significant but also show interesting structures in the crystalline state, as demonstrated herein.<smiles>COC(=O)c1cc(I)cc(C(=O)OC)c1</smiles>

(1)<smiles>C#Cc1cc(C(=O)OC)cc(C(=O)OC)c1</smiles>

(II)

\section{Structural commentary}

The molecular structures of the title compounds, (I) and (II), are illustrated in Fig. $1 a$ and $1 b$, respectively. Taking into account experimental error, the bond distances within the isophthalate framework agree well with those found in the crystal structure of dimethyl isophthalate (Gallagher, 2012). Compound (I) crystallizes in the orthorhombic space group $P n a 2_{1}$ with one molecule in the asymmetric unit. The molecule adopts a twisted conformation with the mean planes defined 

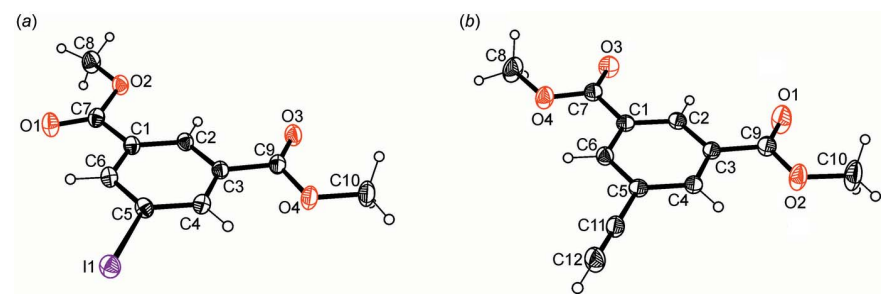

Figure 1

Perspective view of the molecular structures of the title compounds, $(a)$ (I) and (b) (II), with atom labelling. Anisotropic displacement ellipsoids are drawn at the $40 \%$ probability level.

by the methyl carboxylate moieties inclined at angles of $12.6(2)$ and $6.0(2)^{\circ}$ with respect to the plane of the benzene ring. Compound (II) crystallizes in the orthorhombic space group Pnma with the molecule located on a symmetry plane, i.e. the molecule is perfectly planar. However, the molecule adopts approximate $C_{2 v}$ symmetry with the atoms C2, C5, C11 and $\mathrm{C} 12$ lying on a non-crystallographic bisecting symmetry plane.

\section{Supramolecular features}

Infinite strands with the molecules connected via $\mathrm{I} \cdots \mathrm{O}=\mathrm{C}$ interactions [I1 $\cdots \mathrm{O} 3-\mathrm{C} 9\left(x-\frac{1}{2}, y+\frac{3}{2}, z-1 ; D \cdots A=3.129\right.$ (2) (Desiraju \& Steiner, 1999) (Politzer et al. 2007; Desiraju et al., 2013), represent the basic supramolecular aggregates of the crystal structure of (I). Association of the molecular strands by $\mathrm{C}-\mathrm{H} \cdots \mathrm{O}=\mathrm{C}$ type hydrogen bonds (Table 1) (Desiraju \& Steiner, 1999) and $\pi-\pi$ stacking interactions [centroidcentroid distance $=4.149(2) \AA]$ (Tiekink \& ZukermanSchpector, 2012) generate a three-dimensional supramolecular network (Fig. 2). In the crystal structure of (II), the molecules are connected via $\mathrm{C}_{\text {ethynyl }}-\mathrm{H} \cdots \mathrm{O}=\mathrm{C}$ bonds (Table 2) into infinite strands, which are further arranged into molecular sheets that extend parallel to the $a c$ plane (Fig. 3).

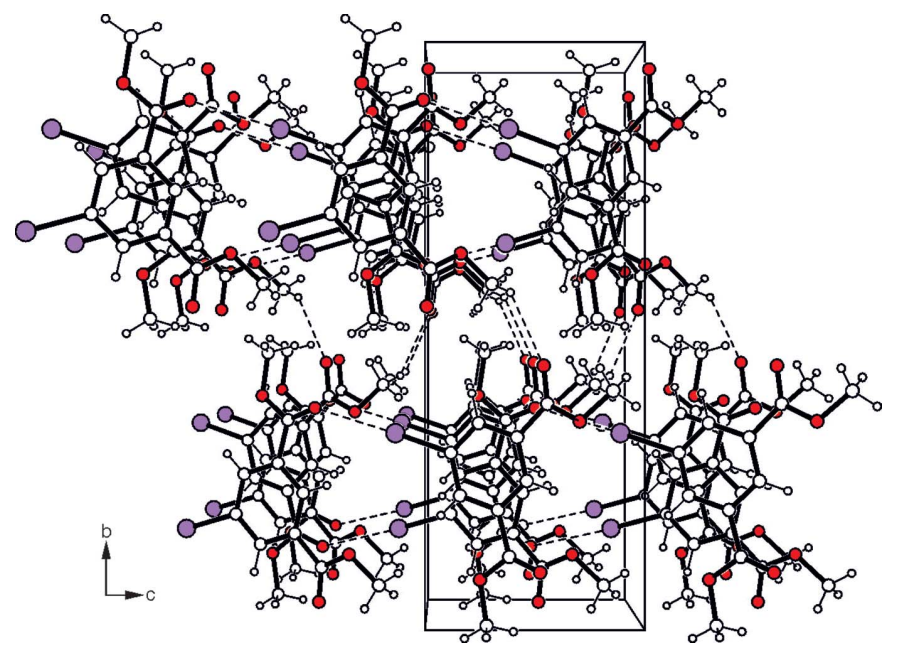

Figure 2

Packing diagram of compound (I) viewed down the $a$ axis. Dashed lines represent hydrogen-bonding interactions.
Table 1

Hydrogen-bond geometry $\left(\AA,^{\circ}\right)$ for (I).

\begin{tabular}{lllll}
\hline$D-\mathrm{H} \cdots A$ & $D-\mathrm{H}$ & $\mathrm{H} \cdots A$ & $D \cdots A$ & $D-\mathrm{H} \cdots A$ \\
\hline $\mathrm{C} 8-\mathrm{H} 8 A \cdots \mathrm{O} 1^{\mathrm{i}}$ & 0.98 & 2.55 & $3.257(4)$ & 129 \\
\hline
\end{tabular}

Symmetry code: (i) $-x+1,-y+1, z-\frac{1}{2}$.

Table 2

Hydrogen-bond geometry ( $\mathrm{A},{ }^{\circ}$ ) for (II).

\begin{tabular}{lllll}
\hline$D-\mathrm{H} \cdots A$ & $D-\mathrm{H}$ & $\mathrm{H} \cdots A$ & $D \cdots A$ & $D-\mathrm{H} \cdots A$ \\
\hline $\mathrm{C} 12-\mathrm{H} 12 \cdots \mathrm{O} 1^{\mathrm{i}}$ & 0.94 & 2.29 & $3.223(1)$ & 172 \\
\hline
\end{tabular}

Symmetry code: (i) $x-1, y, z$.

Furthermore, $\pi-\pi$ arene interactions with a centroid-centroid distance of 3.566 (1) $\AA$ and a slippage of $1.325 \AA$ between the interacting aromatic rings stabilize the crystal structure along the stacking axis of the molecular sheets.

\section{Database survey}

The search in the Cambridge Structural Database (CSD, Version 5.38, update May 2017; Groom et al., 2016) for metasubstituted derivatives of dimethyl isophthalate excluding their metal complexes, solvates and salts gave 18 hits. None of these compounds represents a 5-halogen- and 5-alkynylsubstituted dimethyl isophalate. The parent compound, dimethyl isophthalate (CSD refcode GOHRUS; Gallagher \& Mocilac, 2012) crystallizes in space group $P n a 2_{1}$ with two conformationally similar molecules in the asymmetric unit. The independent molecules participate in different ways in non-covalent bonding. One of them is involved in the formation of linear strands with the molecules connected by $\mathrm{C}-\mathrm{H}_{\text {aryl }} \cdots \mathrm{O}=\mathrm{C}$ bonds. Interstrand association is accomplished by $\pi-\pi$ arene stacking. Molecules related by the twofold screw axis are also linked via $\mathrm{C}-\mathrm{H}_{\text {aryl }} \cdots \mathrm{O}=\mathrm{C}$

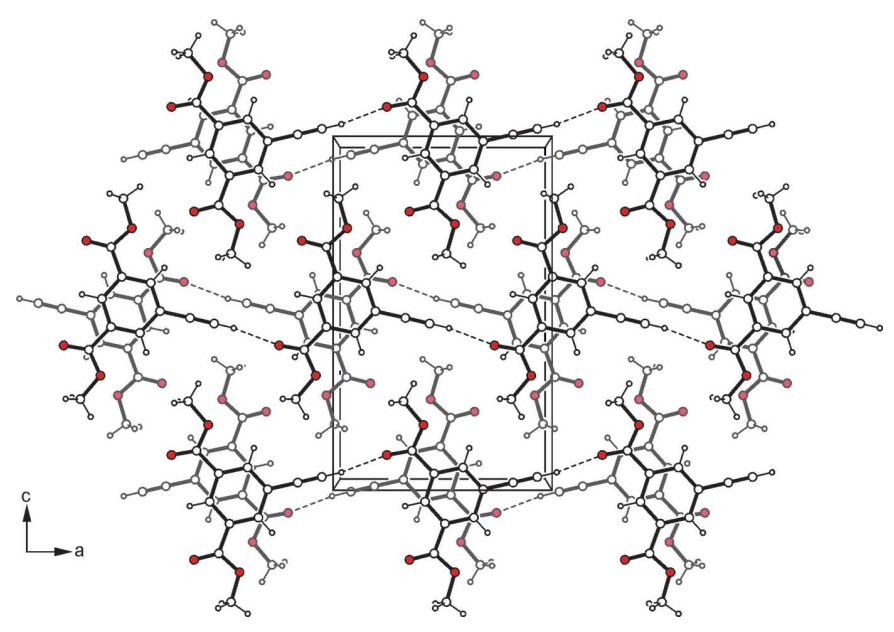

Figure 3

Packing excerpt of compound (II) viewed down the $b$ axis. Dashed lines represent hydrogen-bonding interactions. 
Table 3

Experimental details.

(I)

Crystal data

Chemical formula

$M_{\mathrm{r}}$

Crystal system, space group

Temperature (K)

$a, b, c(\AA)$

$V\left(\AA^{3}\right)$

$Z$

Radiation type

$\mu\left(\mathrm{mm}^{-1}\right)$

Crystal size (mm)

Data collection

Diffractometer

Absorption correction

$T_{\min }, T_{\max }$

No. of measured, independent and

observed $[I>2 \sigma(I)]$ reflections

$R_{\text {int }}$

$(\sin \theta / \lambda)_{\max }\left(\AA^{-1}\right)$
$\mathrm{C}_{10} \mathrm{H}_{9} \mathrm{IO}_{4}$

320.07

Orthorhombic, Pna2

143

7.7483 (2), 19.3451 (6), 7.2338 (2)

$1084.29(5)$

4

Mo $K \alpha$

2.94

$0.30 \times 0.22 \times 0.15$

Bruker APEXII CCD area detector

Multi-scan (SADABS; Sheldrick, 2008a)

$0.472,0.666$

22794, 2909, 2806

0.026

0.684

$0.015,0.038,1.05$

2909

139

1

$\mathrm{H}$-atom parameters constrained

$0.47,-0.44$

Flack $x$ determined using 1255 quotients

$\left[\left(I^{+}\right)-\left(I^{-}\right)\right] /\left[\left(I^{+}\right)+\left(I^{-}\right)\right]$(Parsons et al., 2013)
Absolute structure
(II)

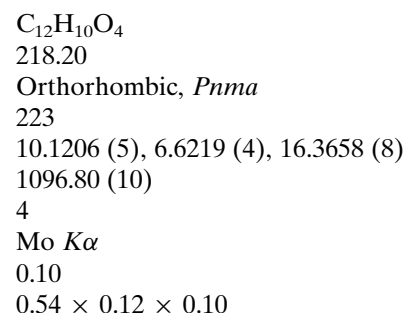

Bruker APEXII CCD area detector Multi-scan (SADABS; Sheldrick, 2008a) $0.948,0.990$

12397, 1292, 932

0.033

0.638

$0.039,0.110,1.03$

1292

87

0

$\mathrm{H}$-atom parameters constrained

$0.17,-0.18$

Absolute structure parameter $-0.004(8)$

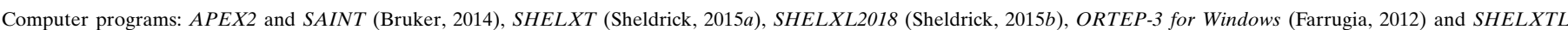
(Sheldrick, 2008b).

bonding to form helical strands. In addition, these strands are stabilized by $\pi-\pi$ stacking forces.

\section{Synthesis and crystallization}

Compounds (I) and (II) were synthesized following literature procedures. This involves a diazotization/iodination reaction of dimethyl 5-aminoisophthalate (Mazik \& König, 2006) to give compound (I). Subsequent reaction of (I) with 2methylbut-3-yne-2-ol (MEBYNOL) using a Pd-catalysed Sonogashira coupling procedure (Doucet \& Hierso, 2007; Rafael \& Carmen, 2007) yielded the corresponding blocked acetylenic diester as an intermediate (Hauptvogel et al., 2011). Removal of the 2-hydroxypropyl blocking group was undertaken using sodium hydride in toluene and quenching with water to result in the title compound (II) (Havens \& Hergenrother, 1985; Hauptvogel et al., 2011).

\section{Refinement}

Crystal data, data collection and structure refinement details are summarized in Table 3. Hydrogen atoms were positioned geometrically and refined using a riding model with $\mathrm{C}-\mathrm{H}$ distances of $0.94-0.98 \AA$ and $U_{\text {iso }}(\mathrm{H})=1.5 U_{\text {eq }}(\mathrm{C}$-methyl $)$ or $U_{\text {iso }}(\mathrm{H})=1.2 U_{\text {eq }}(\mathrm{C})$ for other $\mathrm{H}$ atoms.

\section{Funding information}

We acknowledge the financial support by the Deutsche Forschungsgemeinschaft (DFG Priority Program 1362 'Porous Metal-Organic Frameworks').

\section{References}

Bruker (2014). APEX2 and SAINT. Bruker AXS Inc., Madison, Wisconsin, USA.

Desiraju, G. R., Ho, P. S., Kloo, L., Legon, A. C., Marquardt, R., Metrangolo, P., Politzer, P., Resnati, G. \& Rissanen, K. (2013). Pure Appl. Chem. 85, 1711-1713.

Desiraju, G. R. \& Steiner, T. (1999). In The Weak Hydrogen Bond. Oxford University Press.

Desiraju, G. R., Vittal, J. J. \& Ramanan, A. (2011). Crystal Engineering. Singapore: World Scientific Publications.

Doucet, H. \& Hierso, J. C. (2007). Angew. Chem. Int. Ed. 46, 834871.

Eddaoudi, M., Sava, D. F., Eubank, J. F., Adil, K. \& Guillerm, V. (2015). Chem. Soc. Rev. 44, 228-249.

Farrugia, L. J. (2012). J. Appl. Cryst. 45, 849-854.

Furukawa, H., Cordova, K. E., O'Keeffe, M. \& Yaghi, O. M. (2013). Science, 341, 1230444.

Gallagher, C. F. \& Mocilac, P. (2012). CSD Communication (Refcode GOHRUS). CCDC, Cambridge, England.

Groom, C. R., Bruno, I. J., Lightfoot, M. P. \& Ward, S. C. (2016). Acta Cryst. B72, 171-179. 
Hauptvogel, I. M., Seichter, W. \& Weber, E. (2011). Supramol. Chem. 23, 398-406.

Hausdorf, S., Seichter, W., Weber, E. \& Mertens, F. O. R. L. (2009). Dalton Trans. pp. 1107-1113.

Havens, S. J. \& Hergenrother, P. M. (1985). J. Org. Chem. 50, 18631865.

Lin, X., Jia, J., Zhao, X., Thomas, K. M., Blake, A. J., Walker, G. S., Champness, N. R., Hubberstey, P. \& Schröder, M. (2006). Angew. Chem. Int. Ed. 45, 7358-7364.

MacGillivray, L. R. (2010). Metal-Organic Frameworks. Hoboken: Wiley.

Mazik, M. \& König, A. (2006). J. Org. Chem. 71, 7854-7857.

Parsons, S., Flack, H. D. \& Wagner, T. (2013). Acta Cryst. B69, 249259.
Politzer, P., Lane, P., Concha, M. C., Ma, Y. \& Murray, J. S. (2007). J. Mol. Model. 13, 305-311.

Rafael, C. \& Carmen, N. (2007). Chem. Rev. B107, 874-922.

Sheldrick, G. M. (2008a). SADABS. University of Göttingen, Germany.

Sheldrick, G. M. (2008b). Acta Cryst. A64, 112-122.

Sheldrick, G. M. (2015a). Acta Cryst. A71, 3-8.

Sheldrick, G. M. (2015b). Acta Cryst. C71, 3-8.

Tiekink, E. R. T. \& Zukerman-Schpector, J. (2012). In The Importance of Pi-Interactions in Crystal Engineering. Frontiers in Crystal Engineering. Chichester: Wiley.

Zheng, B., Liang, Z., Li, G., Huo, Q. \& Liu, Y. (2010). Cryst. Growth Des. 10, 3405-3409. 


\section{supporting information}

Acta Cryst. (2018). E74, 1093-1096 [https://doi.org/10.1107/S205698901800912X]

\section{Crystal structures of dimethyl 5-iodoisophthalate and dimethyl 5-}

\section{ethynylisophthalate}

\section{Ines Hauptvogel, Wilhelm Seichter and Edwin Weber}

Computing details

For both structures, data collection: APEX2 (Bruker, 2014); cell refinement: SAINT (Bruker, 2014); data reduction: SAINT (Bruker, 2014); program(s) used to solve structure: SHELXT (Sheldrick, 2015a). Program(s) used to refine structure: SHELXL2018 (Sheldrick, 2015b) for (I); SHELXL2014 (Sheldrick, 2015b) for (II). For both structures, molecular graphics: ORTEP-3 for Windows (Farrugia, 2012); software used to prepare material for publication: SHELXTL (Sheldrick, 2008b).

1,3-Dimethyl 1-iodocyclohexa-3,5-diene-1,3-dicarboxylate (I)

Crystal data

$\mathrm{C}_{10} \mathrm{H}_{9} \mathrm{IO}_{4}$

$M_{r}=320.07$

Orthorhombic, Pna $_{1}$

$a=7.7483(2) \AA$

$b=19.3451(6) \AA$

$c=7.2338(2) \AA$

$V=1084.29(5) \AA^{3}$

$Z=4$

$F(000)=616$

\section{Data collection}

Bruker APEXII CCD area detector diffractometer

$\varphi$ and $\omega$ scans

Absorption correction: multi-scan

(SADABS; Sheldrick, 2008a)

$T_{\text {min }}=0.472, T_{\max }=0.666$

22794 measured reflections

\section{Refinement}

Refinement on $F^{2}$

Least-squares matrix: full

$R\left[F^{2}>2 \sigma\left(F^{2}\right)\right]=0.015$

$w R\left(F^{2}\right)=0.038$

$S=1.05$

2909 reflections

139 parameters

1 restraint

Hydrogen site location: inferred from neighbouring sites
$D_{\mathrm{x}}=1.961 \mathrm{Mg} \mathrm{m}^{-3}$

Mo $K \alpha$ radiation, $\lambda=0.71073 \AA$

Cell parameters from 5755 reflections

$\theta=3.0-33.7^{\circ}$

$\mu=2.94 \mathrm{~mm}^{-1}$

$T=143 \mathrm{~K}$

Irregular, colourless

$0.30 \times 0.22 \times 0.15 \mathrm{~mm}$

2909 independent reflections 2806 reflections with $I>2 \sigma(I)$

$R_{\text {int }}=0.026$

$\theta_{\max }=29.1^{\circ}, \theta_{\min }=1.1^{\circ}$

$h=-10 \rightarrow 10$

$k=-26 \rightarrow 26$

$l=-9 \rightarrow 9$

$\mathrm{H}$-atom parameters constrained

$w=1 /\left[\sigma^{2}\left(F_{0}^{2}\right)+(0.019 P)^{2}+0.3689 P\right]$

where $P=\left(F_{\mathrm{o}}{ }^{2}+2 F_{\mathrm{c}}{ }^{2}\right) / 3$

$(\Delta / \sigma)_{\max }=0.002$

$\Delta \rho_{\max }=0.47 \mathrm{e} \AA^{-3}$

$\Delta \rho_{\min }=-0.44$ e $\AA^{-3}$

Absolute structure: Flack $x$ determined using 1255 quotients $\left[\left(I^{+}\right)-\left(I^{-}\right)\right] /\left[\left(I^{+}\right)+\left(I^{\prime}\right)\right]$ (Parsons et al., 2013)

Absolute structure parameter: -0.004 (8) 


\section{Special details}

Geometry. All esds (except the esd in the dihedral angle between two 1.s. planes) are estimated using the full covariance matrix. The cell esds are taken into account individually in the estimation of esds in distances, angles and torsion angles; correlations between esds in cell parameters are only used when they are defined by crystal symmetry. An approximate (isotropic) treatment of cell esds is used for estimating esds involving l.s. planes.

Refinement. Refined as a 2-component twin.

Fractional atomic coordinates and isotropic or equivalent isotropic displacement parameters $\left(\hat{A}^{2}\right)$

\begin{tabular}{lllll}
\hline & $x$ & $y$ & $z$ & $U_{\text {iso }} / U_{\mathrm{eq}}$ \\
\hline I1 & $0.81504(2)$ & $0.66115(2)$ & $0.83115(6)$ & $0.02386(5)$ \\
O1 & $0.5202(3)$ & $0.54751(11)$ & $0.1864(3)$ & $0.0327(5)$ \\
O2 & $0.3831(3)$ & $0.63417(11)$ & $0.0411(3)$ & $0.0260(4)$ \\
O3 & $0.4971(3)$ & $0.87789(10)$ & $0.2029(3)$ & $0.0281(5)$ \\
O4 & $0.6557(3)$ & $0.89701(11)$ & $0.4576(3)$ & $0.0282(5)$ \\
C1 & $0.5514(4)$ & $0.66267(12)$ & $0.2993(4)$ & $0.0190(8)$ \\
C2 & $0.5347(3)$ & $0.73272(14)$ & $0.2585(4)$ & $0.0198(5)$ \\
H2 & 0.479369 & 0.746965 & 0.147720 & $0.024^{*}$ \\
C3 & $0.5994(3)$ & $0.78152(14)$ & $0.3805(3)$ & $0.0194(5)$ \\
C4 & $0.6796(3)$ & $0.76086(15)$ & $0.5445(4)$ & $0.0206(5)$ \\
H4 & 0.723931 & 0.794439 & 0.627770 & $0.025^{*}$ \\
C5 & $0.6940(3)$ & $0.69088(15)$ & $0.5850(4)$ & $0.0207(5)$ \\
C6 & $0.6295(4)$ & $0.64128(15)$ & $0.4637(4)$ & $0.0209(5)$ \\
H6 & 0.638485 & 0.593502 & 0.492478 & $0.025^{*}$ \\
C7 & $0.4856(4)$ & $0.60819(14)$ & $0.1721(4)$ & $0.0223(5)$ \\
C8 & $0.3221(5)$ & $0.58545(18)$ & $-0.0951(5)$ & $0.0330(7)$ \\
H8A & 0.418544 & 0.571014 & -0.173823 & $0.049^{*}$ \\
H8B & 0.232932 & 0.607288 & -0.171341 & $0.049^{*}$ \\
H8C & 0.273509 & 0.544937 & -0.032593 & $0.049^{*}$ \\
C9 & $0.5768(3)$ & $0.85636(11)$ & $0.3323(8)$ & $0.0214(4)$ \\
C10 & $0.6363(5)$ & $0.97101(16)$ & $0.4293(5)$ & $0.0338(7)$ \\
H10A & 0.688803 & 0.984036 & 0.311034 & $0.051^{*}$ \\
H10B & 0.693716 & 0.995947 & 0.529896 & $0.051^{*}$ \\
H10C & 0.513396 & 0.982919 & 0.427800 & $0.051^{*}$ \\
& & & &
\end{tabular}

Atomic displacement parameters $\left(\AA^{2}\right)$

\begin{tabular}{lllllll}
\hline & $U^{11}$ & $U^{22}$ & $U^{33}$ & $U^{12}$ & $U^{13}$ & $U^{23}$ \\
\hline I1 & $0.02804(8)$ & $0.02336(8)$ & $0.02019(8)$ & $-0.00006(6)$ & $-0.00121(12)$ & $0.00381(11)$ \\
O1 & $0.0500(14)$ & $0.0174(10)$ & $0.0308(12)$ & $0.0032(9)$ & $-0.0060(10)$ & $-0.0006(9)$ \\
O2 & $0.0300(11)$ & $0.0187(10)$ & $0.0293(11)$ & $0.0017(8)$ & $-0.0066(9)$ & $-0.0048(8)$ \\
O3 & $0.0347(12)$ & $0.0194(10)$ & $0.0301(11)$ & $0.0027(9)$ & $-0.0082(9)$ & $0.0019(8)$ \\
O4 & $0.0382(12)$ & $0.0165(9)$ & $0.0299(11)$ & $-0.0011(8)$ & $-0.0073(9)$ & $-0.0004(9)$ \\
C1 & $0.0216(11)$ & $0.0183(10)$ & $0.017(2)$ & $0.0009(9)$ & $0.0020(10)$ & $-0.0004(9)$ \\
C2 & $0.0196(12)$ & $0.0190(12)$ & $0.0209(11)$ & $0.0022(10)$ & $0.0042(10)$ & $0.0019(10)$ \\
C3 & $0.0191(11)$ & $0.0183(11)$ & $0.0208(13)$ & $0.0013(9)$ & $0.0025(9)$ & $0.0012(8)$ \\
C4 & $0.0217(13)$ & $0.0191(13)$ & $0.0211(12)$ & $-0.0014(10)$ & $0.0017(10)$ & $-0.0012(10)$ \\
C5 & $0.0224(13)$ & $0.0217(13)$ & $0.0181(12)$ & $0.0022(10)$ & $0.0023(10)$ & $0.0017(10)$
\end{tabular}




\begin{tabular}{lllllll} 
C6 & $0.0242(13)$ & $0.0183(12)$ & $0.0201(12)$ & $0.0016(10)$ & $0.0024(11)$ & $0.0013(10)$ \\
C7 & $0.0256(13)$ & $0.0197(12)$ & $0.0217(13)$ & $-0.0011(10)$ & $0.0035(11)$ & $-0.0011(10)$ \\
C8 & $0.0409(19)$ & $0.0257(15)$ & $0.0323(15)$ & $0.0005(13)$ & $-0.0088(13)$ & $-0.0075(13)$ \\
C9 & $0.0218(10)$ & $0.0173(9)$ & $0.0250(10)$ & $-0.0001(8)$ & $0.0096(18)$ & $-0.002(2)$ \\
C10 & $0.0453(19)$ & $0.0174(14)$ & $0.0387(19)$ & $0.0014(13)$ & $-0.0064(15)$ & $-0.0021(12)$ \\
\hline
\end{tabular}

Geometric parameters $\left(\hat{A},{ }^{\circ}\right)$

\begin{tabular}{|c|c|c|c|}
\hline $\mathrm{I} 1-\mathrm{C} 5$ & $2.093(3)$ & $\mathrm{C} 3-\mathrm{C} 4$ & $1.398(4)$ \\
\hline $\mathrm{O} 1-\mathrm{C} 7$ & $1.209(3)$ & $\mathrm{C} 3-\mathrm{C} 9$ & $1.499(4)$ \\
\hline $\mathrm{O} 2-\mathrm{C} 7$ & $1.335(4)$ & $\mathrm{C} 4-\mathrm{C} 5$ & $1.390(4)$ \\
\hline $\mathrm{O} 2-\mathrm{C} 8$ & $1.443(4)$ & $\mathrm{C} 4-\mathrm{H} 4$ & 0.9500 \\
\hline $\mathrm{O} 3-\mathrm{C} 9$ & $1.196(5)$ & $\mathrm{C} 5-\mathrm{C} 6$ & $1.393(4)$ \\
\hline $\mathrm{O} 4-\mathrm{C} 9$ & $1.347(5)$ & $\mathrm{C} 6-\mathrm{H} 6$ & 0.9500 \\
\hline $\mathrm{O} 4-\mathrm{C} 10$ & $1.454(4)$ & $\mathrm{C} 8-\mathrm{H} 8 \mathrm{~A}$ & 0.9800 \\
\hline $\mathrm{C} 1-\mathrm{C} 2$ & $1.393(3)$ & $\mathrm{C} 8-\mathrm{H} 8 \mathrm{~B}$ & 0.9800 \\
\hline $\mathrm{C} 1-\mathrm{C} 6$ & $1.398(4)$ & $\mathrm{C} 8-\mathrm{H} 8 \mathrm{C}$ & 0.9800 \\
\hline $\mathrm{C} 1-\mathrm{C} 7$ & $1.489(4)$ & $\mathrm{C} 10-\mathrm{H} 10 \mathrm{~A}$ & 0.9800 \\
\hline $\mathrm{C} 2-\mathrm{C} 3$ & $1.386(4)$ & $\mathrm{C} 10-\mathrm{H} 10 \mathrm{~B}$ & 0.9800 \\
\hline $\mathrm{C} 2-\mathrm{H} 2$ & 0.9500 & $\mathrm{C} 10-\mathrm{H} 10 \mathrm{C}$ & 0.9800 \\
\hline $\mathrm{C} 7-\mathrm{O} 2-\mathrm{C} 8$ & $115.7(2)$ & $\mathrm{C} 1-\mathrm{C} 6-\mathrm{H} 6$ & 120.4 \\
\hline $\mathrm{C} 9-\mathrm{O} 4-\mathrm{C} 10$ & $115.7(3)$ & $\mathrm{O} 1-\mathrm{C} 7-\mathrm{O} 2$ & $124.0(3)$ \\
\hline $\mathrm{C} 2-\mathrm{C} 1-\mathrm{C} 6$ & $120.5(3)$ & $\mathrm{O} 1-\mathrm{C} 7-\mathrm{C} 1$ & $124.0(3)$ \\
\hline $\mathrm{C} 2-\mathrm{C} 1-\mathrm{C} 7$ & $121.8(3)$ & $\mathrm{O} 2-\mathrm{C} 7-\mathrm{C} 1$ & $112.1(2)$ \\
\hline $\mathrm{C} 6-\mathrm{C} 1-\mathrm{C} 7$ & $117.7(2)$ & $\mathrm{O} 2-\mathrm{C} 8-\mathrm{H} 8 \mathrm{~A}$ & 109.5 \\
\hline $\mathrm{C} 3-\mathrm{C} 2-\mathrm{C} 1$ & $119.7(3)$ & $\mathrm{O} 2-\mathrm{C} 8-\mathrm{H} 8 \mathrm{~B}$ & 109.5 \\
\hline $\mathrm{C} 3-\mathrm{C} 2-\mathrm{H} 2$ & 120.2 & $\mathrm{H} 8 \mathrm{~A}-\mathrm{C} 8-\mathrm{H} 8 \mathrm{~B}$ & 109.5 \\
\hline $\mathrm{C} 1-\mathrm{C} 2-\mathrm{H} 2$ & 120.2 & $\mathrm{O} 2-\mathrm{C} 8-\mathrm{H} 8 \mathrm{C}$ & 109.5 \\
\hline $\mathrm{C} 2-\mathrm{C} 3-\mathrm{C} 4$ & $120.4(3)$ & $\mathrm{H} 8 \mathrm{~A}-\mathrm{C} 8-\mathrm{H} 8 \mathrm{C}$ & 109.5 \\
\hline $\mathrm{C} 2-\mathrm{C} 3-\mathrm{C} 9$ & $117.9(3)$ & $\mathrm{H} 8 \mathrm{~B}-\mathrm{C} 8-\mathrm{H} 8 \mathrm{C}$ & 109.5 \\
\hline $\mathrm{C} 4-\mathrm{C} 3-\mathrm{C} 9$ & $121.7(3)$ & $\mathrm{O} 3-\mathrm{C} 9-\mathrm{O} 4$ & $123.9(2)$ \\
\hline $\mathrm{C} 5-\mathrm{C} 4-\mathrm{C} 3$ & $119.5(3)$ & $\mathrm{O} 3-\mathrm{C} 9-\mathrm{C} 3$ & $125.3(3)$ \\
\hline $\mathrm{C} 5-\mathrm{C} 4-\mathrm{H} 4$ & 120.2 & $\mathrm{O} 4-\mathrm{C} 9-\mathrm{C} 3$ & $110.7(3)$ \\
\hline $\mathrm{C} 3-\mathrm{C} 4-\mathrm{H} 4$ & 120.2 & $\mathrm{O} 4-\mathrm{C} 10-\mathrm{H} 10 \mathrm{~A}$ & 109.5 \\
\hline $\mathrm{C} 4-\mathrm{C} 5-\mathrm{C} 6$ & $120.6(3)$ & $\mathrm{O} 4-\mathrm{C} 10-\mathrm{H} 10 \mathrm{~B}$ & 109.5 \\
\hline $\mathrm{C} 4-\mathrm{C} 5-\mathrm{I} 1$ & $118.9(2)$ & $\mathrm{H} 10 \mathrm{~A}-\mathrm{C} 10-\mathrm{H} 10 \mathrm{~B}$ & 109.5 \\
\hline $\mathrm{C} 6-\mathrm{C} 5-\mathrm{I} 1$ & $120.5(2)$ & $\mathrm{O} 4-\mathrm{C} 10-\mathrm{H} 10 \mathrm{C}$ & 109.5 \\
\hline $\mathrm{C} 5-\mathrm{C} 6-\mathrm{C} 1$ & $119.2(3)$ & $\mathrm{H} 10 \mathrm{~A}-\mathrm{C} 10-\mathrm{H} 10 \mathrm{C}$ & 109.5 \\
\hline $\mathrm{C} 5-\mathrm{C} 6-\mathrm{H} 6$ & 120.4 & $\mathrm{H} 10 \mathrm{~B}-\mathrm{C} 10-\mathrm{H} 10 \mathrm{C}$ & 109.5 \\
\hline $\mathrm{C} 6-\mathrm{C} 1-\mathrm{C} 2-\mathrm{C} 3$ & $1.3(4)$ & $\mathrm{C} 8-\mathrm{O} 2-\mathrm{C} 7-\mathrm{O} 1$ & $-4.1(4)$ \\
\hline $\mathrm{C} 7-\mathrm{C} 1-\mathrm{C} 2-\mathrm{C} 3$ & $-179.4(2)$ & $\mathrm{C} 8-\mathrm{O} 2-\mathrm{C} 7-\mathrm{C} 1$ & $176.3(3)$ \\
\hline $\mathrm{C} 1-\mathrm{C} 2-\mathrm{C} 3-\mathrm{C} 4$ & $-0.6(4)$ & $\mathrm{C} 2-\mathrm{C} 1-\mathrm{C} 7-\mathrm{O} 1$ & $168.0(3)$ \\
\hline $\mathrm{C} 1-\mathrm{C} 2-\mathrm{C} 3-\mathrm{C} 9$ & $-179.3(3)$ & $\mathrm{C} 6-\mathrm{C} 1-\mathrm{C} 7-\mathrm{O} 1$ & $-12.7(4)$ \\
\hline $\mathrm{C} 2-\mathrm{C} 3-\mathrm{C} 4-\mathrm{C} 5$ & $-0.1(4)$ & $\mathrm{C} 2-\mathrm{C} 1-\mathrm{C} 7-\mathrm{O} 2$ & $-12.5(4)$ \\
\hline $\mathrm{C} 9-\mathrm{C} 3-\mathrm{C} 4-\mathrm{C} 5$ & 178.5 & $\mathrm{C} 6-\mathrm{C} 1-\mathrm{C} 7-\mathrm{O} 2$ & $166.8(3)$ \\
\hline $\mathrm{C} 3-\mathrm{C} 4-\mathrm{C} 5-\mathrm{C} 6$ & $0.1(4)$ & $\mathrm{C} 10-\mathrm{O} 4-\mathrm{C} 9-\mathrm{O} 3$ & $1.1(5)$ \\
\hline
\end{tabular}




$\begin{array}{llll}\mathrm{C} 3-\mathrm{C} 4-\mathrm{C} 5-\mathrm{I} 1 & 179.86(19) & \mathrm{C} 10-\mathrm{O} 4-\mathrm{C} 9-\mathrm{C} 3 & -177.5(3) \\ \mathrm{C} 4-\mathrm{C} 5-\mathrm{C} 6-\mathrm{C} 1 & 0.7(4) & \mathrm{C} 2-\mathrm{C} 3-\mathrm{C} 9-\mathrm{O} 3 & 5.3(5) \\ \mathrm{I} 1-\mathrm{C} 5-\mathrm{C} 6-\mathrm{C} 1 & -179.1(2) & \mathrm{C} 4-\mathrm{C} 3-\mathrm{C} 9-\mathrm{O} 3 & -173.3(3) \\ \mathrm{C} 2-\mathrm{C} 1-\mathrm{C} 6-\mathrm{C} 5 & -1.3(4) & \mathrm{C} 2-\mathrm{C} 3-\mathrm{C} 9-\mathrm{O} 4 & -176.1(3) \\ \mathrm{C} 7-\mathrm{C} 1-\mathrm{C} 6-\mathrm{C} 5 & 179.4(2) & \mathrm{C} 4-\mathrm{C} 3-\mathrm{C} 9-\mathrm{O} 4 & 5.3(4)\end{array}$

Hydrogen-bond geometry $\left(\AA,{ }^{\circ}\right)$

\begin{tabular}{lllll}
\hline$D-\mathrm{H} \cdots A$ & $D-\mathrm{H}$ & $\mathrm{H} \cdots A$ & $D \cdots A$ & $D-\mathrm{H} \cdots A$ \\
\hline $\mathrm{C} 8-\mathrm{H} 8 A \cdots \mathrm{O} 1^{\mathrm{i}}$ & 0.98 & 2.55 & $3.257(4)$ & 129 \\
\hline
\end{tabular}

Symmetry code: (i) $-x+1,-y+1, z-1 / 2$.

1,3-Dimethyl 1-ethynylcyclohexa-3,5-diene-1,3-dicarboxylate (II)

Crystal data

$\mathrm{C}_{12} \mathrm{H}_{10} \mathrm{O}_{4}$

$M_{r}=218.20$

Orthorhombic, Pnma

$a=10.1206$ (5) $\AA$

$b=6.6219$ (4) $\AA$

$c=16.3658(8) \AA$

$V=1096.80(10) \AA^{3}$

$Z=4$

$F(000)=456$

\section{Data collection}

Bruker APEXII CCD area detector diffractometer

$\varphi$ and $\omega$ scans

Absorption correction: multi-scan

(SADABS; Sheldrick, 2008a)

$T_{\min }=0.948, T_{\max }=0.990$

12397 measured reflections

\section{Refinement}

Refinement on $F^{2}$

Least-squares matrix: full

$R\left[F^{2}>2 \sigma\left(F^{2}\right)\right]=0.039$

$w R\left(F^{2}\right)=0.110$

$S=1.03$

1292 reflections

87 parameters

0 restraints
$D_{\mathrm{x}}=1.321 \mathrm{Mg} \mathrm{m}^{-3}$

Mo $K \alpha$ radiation, $\lambda=0.71073 \AA$

Cell parameters from 2950 reflections

$\theta=2.4-23.1^{\circ}$

$\mu=0.10 \mathrm{~mm}^{-1}$

$T=223 \mathrm{~K}$

Column, colourless

$0.54 \times 0.12 \times 0.10 \mathrm{~mm}$

1292 independent reflections 932 reflections with $I>2 \sigma(I)$

$R_{\text {int }}=0.033$

$\theta_{\max }=27.0^{\circ}, \theta_{\min }=2.5^{\circ}$

$h=-12 \rightarrow 12$

$k=-8 \rightarrow 5$

$l=-20 \rightarrow 19$

Hydrogen site location: inferred from neighbouring sites

$\mathrm{H}$-atom parameters constrained

$w=1 /\left[\sigma^{2}\left(F_{\mathrm{o}}^{2}\right)+(0.0486 P)^{2}+0.2932 P\right]$

where $P=\left(F_{\mathrm{o}}^{2}+2 F_{\mathrm{c}}^{2}\right) / 3$

$(\Delta / \sigma)_{\max }<0.001$

$\Delta \rho_{\max }=0.17 \mathrm{e} \AA^{-3}$

$\Delta \rho_{\min }=-0.18$ e $\AA^{-3}$

Special details

Geometry. All esds (except the esd in the dihedral angle between two 1.s. planes) are estimated using the full covariance matrix. The cell esds are taken into account individually in the estimation of esds in distances, angles and torsion angles; correlations between esds in cell parameters are only used when they are defined by crystal symmetry. An approximate (isotropic) treatment of cell esds is used for estimating esds involving l.s. planes. 
Fractional atomic coordinates and isotropic or equivalent isotropic displacement parameters $\left(\hat{A}^{2}\right)$

\begin{tabular}{llllll}
\hline & $x$ & $y$ & $z$ & $U_{\text {iso }} * / U_{\text {eq }}$ & Occ. $(<1)$ \\
\hline O1 & $1.25864(13)$ & 0.2500 & $0.40733(10)$ & $0.0552(4)$ & \\
O2 & $1.08848(14)$ & 0.2500 & $0.32081(9)$ & $0.0519(4)$ & \\
O3 & $1.15356(16)$ & 0.2500 & $0.70802(10)$ & $0.0759(6)$ & \\
O4 & $0.94247(15)$ & 0.2500 & $0.74402(9)$ & $0.0617(5)$ & \\
C1 & $0.98919(18)$ & 0.2500 & $0.60451(11)$ & $0.0339(4)$ & \\
C2 & $1.08100(18)$ & 0.2500 & $0.54143(12)$ & $0.0350(4)$ & \\
H2 & 1.1718 & 0.2500 & 0.5536 & $0.042^{*}$ & \\
C3 & $1.03958(18)$ & 0.2500 & $0.46063(12)$ & $0.0339(4)$ & \\
C4 & $0.90505(19)$ & 0.2500 & $0.44325(12)$ & $0.0361(4)$ & \\
H4 & 0.8767 & 0.2500 & 0.3886 & $0.043 *$ & \\
C5 & $0.81192(17)$ & 0.2500 & $0.50582(12)$ & $0.0348(4)$ & \\
C6 & $0.85496(18)$ & 0.2500 & $0.58682(12)$ & $0.0340(4)$ & \\
H6 & 0.7929 & 0.2500 & 0.6296 & $0.041^{*}$ & \\
C7 & $1.0392(2)$ & 0.2500 & $0.68995(13)$ & $0.0428(5)$ & \\
C8 & $0.9818(3)$ & 0.2500 & $0.82935(14)$ & $0.0791(9)$ & \\
H8A & 1.0203 & 0.1202 & 0.8431 & $0.119^{*}$ & \\
H8B & 0.9050 & 0.2740 & 0.8634 & $0.119^{*}$ & 0.5 \\
H8C & 1.0465 & 0.3558 & 0.8385 & $0.119^{*}$ & \\
C9 & 1.1418 & 0.2500 & 0.3949 & 0.039 & \\
C10 & 1.1813 & 0.2500 & 0.2530 & 0.067 & 0.5 \\
H10A & 1.1353 & 0.2149 & 0.2030 & $0.101^{*}$ & \\
H10B & 1.2505 & 0.1519 & 0.2633 & $0.101^{*}$ & \\
H10C & 1.2202 & 0.3832 & 0.2474 & $0.101^{*}$ & 0.5 \\
C11 & $0.67260(19)$ & 0.2500 & $0.48649(12)$ & $0.0406(5)$ & \\
C12 & $0.5604(2)$ & 0.2500 & $0.47022(14)$ & $0.0532(6)$ & \\
H12 & 0.4700 & 0.2500 & 0.4571 & $0.064 *$ & \\
& & & & & \\
& & & & \\
\end{tabular}

Atomic displacement parameters $\left(\AA^{2}\right)$

\begin{tabular}{|c|c|c|c|c|c|c|}
\hline & $U^{11}$ & $U^{22}$ & $U^{33}$ & $U^{12}$ & $U^{13}$ & $U^{23}$ \\
\hline O1 & $0.0293(8)$ & $0.0828(11)$ & $0.0536(10)$ & 0.000 & $0.0049(7)$ & 0.000 \\
\hline $\mathrm{O} 2$ & $0.0409(9)$ & $0.0783(11)$ & $0.0364(8)$ & 0.000 & $0.0066(7)$ & 0.000 \\
\hline $\mathrm{O} 3$ & $0.0329(9)$ & $0.1475(18)$ & $0.0473(10)$ & 0.000 & $-0.0079(8)$ & 0.000 \\
\hline $\mathrm{O} 4$ & $0.0362(9)$ & 0.1153 (14) & $0.0336(8)$ & 0.000 & $-0.0026(7)$ & 0.000 \\
\hline $\mathrm{C} 1$ & $0.0294(10)$ & $0.0371(10)$ & $0.0352(11)$ & 0.000 & $-0.0006(8)$ & 0.000 \\
\hline $\mathrm{C} 2$ & $0.0257(9)$ & $0.0385(10)$ & $0.0408(11)$ & 0.000 & $-0.0029(8)$ & 0.000 \\
\hline $\mathrm{C} 3$ & $0.0295(10)$ & $0.0338(9)$ & $0.0385(11)$ & 0.000 & $0.0023(8)$ & 0.000 \\
\hline $\mathrm{C} 4$ & $0.0343(11)$ & $0.0404(10)$ & $0.0334(10)$ & 0.000 & $-0.0020(9)$ & 0.000 \\
\hline $\mathrm{C} 5$ & $0.0275(9)$ & $0.0379(10)$ & $0.0389(11)$ & 0.000 & $-0.0002(8)$ & 0.000 \\
\hline C6 & $0.0276(9)$ & $0.0398(10)$ & $0.0347(11)$ & 0.000 & $0.0011(8)$ & 0.000 \\
\hline $\mathrm{C} 7$ & $0.0299(11)$ & $0.0568(12)$ & $0.0416(12)$ & 0.000 & $-0.0013(9)$ & 0.000 \\
\hline $\mathrm{C} 8$ & $0.0550(16)$ & $0.151(3)$ & $0.0315(13)$ & 0.000 & $-0.0049(12)$ & 0.000 \\
\hline C9 & 0.034 & 0.043 & 0.039 & 0.000 & 0.003 & 0.000 \\
\hline $\mathrm{C} 10$ & 0.063 & 0.098 & 0.041 & 0.000 & 0.018 & 0.000 \\
\hline $\mathrm{C} 11$ & $0.0341(11)$ & $0.0558(12)$ & $0.0319(10)$ & 0.000 & $0.0003(9)$ & 0.000 \\
\hline
\end{tabular}


Geometric parameters $\left(\AA,{ }^{\circ}\right)$

\begin{tabular}{|c|c|c|c|}
\hline $\mathrm{O} 1-\mathrm{C} 9$ & $1.1998(14)$ & $\mathrm{C} 4-\mathrm{C} 5$ & $1.392(3)$ \\
\hline $\mathrm{O} 2-\mathrm{C} 9$ & $1.3272(15)$ & $\mathrm{C} 4-\mathrm{H} 4$ & 0.9400 \\
\hline $\mathrm{O} 2-\mathrm{C} 10$ & $1.4544(14)$ & $\mathrm{C} 5-\mathrm{C} 6$ & $1.395(3)$ \\
\hline $\mathrm{O} 3-\mathrm{C} 7$ & $1.195(3)$ & $\mathrm{C} 5-\mathrm{C} 11$ & $1.445(3)$ \\
\hline $\mathrm{O} 4-\mathrm{C} 7$ & $1.319(3)$ & $\mathrm{C} 6-\mathrm{H} 6$ & 0.9400 \\
\hline $\mathrm{O} 4-\mathrm{C} 8$ & $1.452(3)$ & $\mathrm{C} 8-\mathrm{H} 8 \mathrm{~A}$ & 0.9700 \\
\hline $\mathrm{C} 1-\mathrm{C} 2$ & $1.389(3)$ & $\mathrm{C} 8-\mathrm{H} 8 \mathrm{~B}$ & 0.9700 \\
\hline $\mathrm{C} 1-\mathrm{C} 6$ & $1.389(3)$ & $\mathrm{C} 8-\mathrm{H} 8 \mathrm{C}$ & 0.9700 \\
\hline $\mathrm{C} 1-\mathrm{C} 7$ & $1.487(3)$ & $\mathrm{C} 10-\mathrm{H} 10 \mathrm{~A}$ & 0.9700 \\
\hline $\mathrm{C} 2-\mathrm{C} 3$ & $1.387(3)$ & $\mathrm{C} 10-\mathrm{H} 10 \mathrm{~B}$ & 0.9700 \\
\hline $\mathrm{C} 2-\mathrm{H} 2$ & 0.9400 & $\mathrm{C} 10-\mathrm{H} 10 \mathrm{C}$ & 0.9700 \\
\hline $\mathrm{C} 3-\mathrm{C} 4$ & $1.391(3)$ & $\mathrm{C} 11-\mathrm{C} 12$ & $1.166(3)$ \\
\hline $\mathrm{C} 3-\mathrm{C} 9$ & $1.4925(18)$ & $\mathrm{C} 12-\mathrm{H} 12$ & 0.9400 \\
\hline $\mathrm{C} 9-\mathrm{O} 2-\mathrm{C} 10$ & $115.75(10)$ & $\mathrm{O} 3-\mathrm{C} 7-\mathrm{O} 4$ & $123.6(2)$ \\
\hline $\mathrm{C} 7-\mathrm{O} 4-\mathrm{C} 8$ & $116.19(18)$ & $\mathrm{O} 3-\mathrm{C} 7-\mathrm{C} 1$ & $124.21(19)$ \\
\hline $\mathrm{C} 2-\mathrm{C} 1-\mathrm{C} 6$ & $119.96(18)$ & $\mathrm{O} 4-\mathrm{C} 7-\mathrm{C} 1$ & $112.23(17)$ \\
\hline $\mathrm{C} 2-\mathrm{C} 1-\mathrm{C} 7$ & $118.13(17)$ & $\mathrm{O} 4-\mathrm{C} 8-\mathrm{H} 8 \mathrm{~A}$ & 109.5 \\
\hline $\mathrm{C} 6-\mathrm{C} 1-\mathrm{C} 7$ & $121.91(17)$ & $\mathrm{O} 4-\mathrm{C} 8-\mathrm{H} 8 \mathrm{~B}$ & 109.5 \\
\hline $\mathrm{C} 3-\mathrm{C} 2-\mathrm{C} 1$ & $120.42(17)$ & $\mathrm{H} 8 \mathrm{~A}-\mathrm{C} 8-\mathrm{H} 8 \mathrm{~B}$ & 109.5 \\
\hline $\mathrm{C} 3-\mathrm{C} 2-\mathrm{H} 2$ & 119.8 & $\mathrm{O} 4-\mathrm{C} 8-\mathrm{H} 8 \mathrm{C}$ & 109.5 \\
\hline $\mathrm{C} 1-\mathrm{C} 2-\mathrm{H} 2$ & 119.8 & $\mathrm{H} 8 \mathrm{~A}-\mathrm{C} 8-\mathrm{H} 8 \mathrm{C}$ & 109.5 \\
\hline $\mathrm{C} 2-\mathrm{C} 3-\mathrm{C} 4$ & $119.39(18)$ & $\mathrm{H} 8 \mathrm{~B}-\mathrm{C} 8-\mathrm{H} 8 \mathrm{C}$ & 109.5 \\
\hline $\mathrm{C} 2-\mathrm{C} 3-\mathrm{C} 9$ & $118.53(15)$ & $\mathrm{O} 1-\mathrm{C} 9-\mathrm{O} 2$ & $123.76(10)$ \\
\hline $\mathrm{C} 4-\mathrm{C} 3-\mathrm{C} 9$ & $122.09(16)$ & $\mathrm{O} 1-\mathrm{C} 9-\mathrm{C} 3$ & $124.12(11)$ \\
\hline $\mathrm{C} 3-\mathrm{C} 4-\mathrm{C} 5$ & $120.83(18)$ & $\mathrm{O} 2-\mathrm{C} 9-\mathrm{C} 3$ & $112.12(9)$ \\
\hline $\mathrm{C} 3-\mathrm{C} 4-\mathrm{H} 4$ & 119.6 & $\mathrm{O} 2-\mathrm{C} 10-\mathrm{H} 10 \mathrm{~A}$ & 109.5 \\
\hline $\mathrm{C} 5-\mathrm{C} 4-\mathrm{H} 4$ & 119.6 & $\mathrm{O} 2-\mathrm{C} 10-\mathrm{H} 10 \mathrm{~B}$ & 109.5 \\
\hline $\mathrm{C} 4-\mathrm{C} 5-\mathrm{C} 6$ & $119.18(17)$ & $\mathrm{H} 10 \mathrm{~A}-\mathrm{C} 10-\mathrm{H} 10 \mathrm{~B}$ & 109.5 \\
\hline $\mathrm{C} 4-\mathrm{C} 5-\mathrm{C} 11$ & $119.98(18)$ & $\mathrm{O} 2-\mathrm{C} 10-\mathrm{H} 10 \mathrm{C}$ & 109.5 \\
\hline $\mathrm{C} 6-\mathrm{C} 5-\mathrm{C} 11$ & $120.84(17)$ & $\mathrm{H} 10 \mathrm{~A}-\mathrm{C} 10-\mathrm{H} 10 \mathrm{C}$ & 109.5 \\
\hline $\mathrm{C} 1-\mathrm{C} 6-\mathrm{C} 5$ & $120.21(17)$ & $\mathrm{H} 10 \mathrm{~B}-\mathrm{C} 10-\mathrm{H} 10 \mathrm{C}$ & 109.5 \\
\hline $\mathrm{C} 1-\mathrm{C} 6-\mathrm{H} 6$ & 119.9 & $\mathrm{C} 12-\mathrm{C} 11-\mathrm{C} 5$ & $179.5(2)$ \\
\hline $\mathrm{C} 5-\mathrm{C} 6-\mathrm{H} 6$ & 119.9 & $\mathrm{C} 11-\mathrm{C} 12-\mathrm{H} 12$ & 180.0 \\
\hline $\mathrm{C} 6-\mathrm{C} 1-\mathrm{C} 2-\mathrm{C} 3$ & $0.000(1)$ & $\mathrm{C} 8-\mathrm{O} 4-\mathrm{C} 7-\mathrm{O} 3$ & $0.000(1)$ \\
\hline $\mathrm{C} 7-\mathrm{C} 1-\mathrm{C} 2-\mathrm{C} 3$ & $180.000(1)$ & $\mathrm{C} 8-\mathrm{O} 4-\mathrm{C} 7-\mathrm{C} 1$ & $180.000(1)$ \\
\hline $\mathrm{C} 1-\mathrm{C} 2-\mathrm{C} 3-\mathrm{C} 4$ & 0.000 & $\mathrm{C} 2-\mathrm{C} 1-\mathrm{C} 7-\mathrm{O} 3$ & 0.000 \\
\hline $\mathrm{C} 1-\mathrm{C} 2-\mathrm{C} 3-\mathrm{C} 9$ & $180.000(1)$ & $\mathrm{C} 6-\mathrm{C} 1-\mathrm{C} 7-\mathrm{O} 3$ & $180.000(1)$ \\
\hline $\mathrm{C} 2-\mathrm{C} 3-\mathrm{C} 4-\mathrm{C} 5$ & $0.000(1)$ & $\mathrm{C} 2-\mathrm{C} 1-\mathrm{C} 7-\mathrm{O} 4$ & $180.000(1)$ \\
\hline $\mathrm{C} 9-\mathrm{C} 3-\mathrm{C} 4-\mathrm{C} 5$ & $180.000(1)$ & $\mathrm{C} 6-\mathrm{C} 1-\mathrm{C} 7-\mathrm{O} 4$ & $0.000(1)$ \\
\hline $\mathrm{C} 3-\mathrm{C} 4-\mathrm{C} 5-\mathrm{C} 6$ & 0.000 & $\mathrm{C} 10-\mathrm{O} 2-\mathrm{C} 9-\mathrm{O} 1$ & 0.000 \\
\hline $\mathrm{C} 3-\mathrm{C} 4-\mathrm{C} 5-\mathrm{C} 11$ & $180.000(1)$ & $\mathrm{C} 10-\mathrm{O} 2-\mathrm{C} 9-\mathrm{C} 3$ & $180.000(1)$ \\
\hline $\mathrm{C} 2-\mathrm{C} 1-\mathrm{C} 6-\mathrm{C} 5$ & 0.000 & $\mathrm{C} 2-\mathrm{C} 3-\mathrm{C} 9-\mathrm{O} 1$ & 0.000 \\
\hline
\end{tabular}


supporting information

\begin{tabular}{llll}
$\mathrm{C} 7-\mathrm{C} 1-\mathrm{C} 6-\mathrm{C} 5$ & $180.000(1)$ & $\mathrm{C} 4-\mathrm{C} 3-\mathrm{C} 9-\mathrm{O} 1$ & $180.000(1)$ \\
$\mathrm{C} 4-\mathrm{C} 5-\mathrm{C} 6-\mathrm{C} 1$ & $0.000(1)$ & $\mathrm{C} 2-\mathrm{C} 3-\mathrm{C} 9-\mathrm{O} 2$ & $180.000(1)$ \\
$\mathrm{C} 11-\mathrm{C} 5-\mathrm{C} 6-\mathrm{C} 1$ & $180.000(1)$ & $\mathrm{C} 4-\mathrm{C} 3-\mathrm{C} 9-\mathrm{O} 2$ & $0.000(1)$ \\
\hline
\end{tabular}

Hydrogen-bond geometry $\left(A,{ }^{\circ}\right)$

\begin{tabular}{lllll}
\hline$D-\mathrm{H} \cdots A$ & $D-\mathrm{H}$ & $\mathrm{H} \cdots A$ & $D \cdots A$ & $D-\mathrm{H} \cdots A$ \\
\hline $\mathrm{C} 12-\mathrm{H} 12 \cdots \mathrm{O} 1^{\mathrm{i}}$ & 0.94 & 2.29 & $3.223(1)$ & 172 \\
\hline
\end{tabular}

Symmetry code: (i) $x-1, y, z$. 